Bull. Austral. Math. Soc.

VoL. 46 (1992) [441-448]

\title{
THE STABILITY OF SOLUTIONS \\ IN AN INITIAL-BOUNDARY REACTION-DIFFUSION SYSTEM
}

\author{
E. Tuma ANd C.M. BlázQUez
}

We study the asymptotic behaviour as $t \rightarrow \infty$ of solutions of the initial-boundary value problem $v_{t}=G(u, v), u_{t}=u_{x x}+F(u, v)$, and $t>0, x \in \mathbb{R}$ or $x \in \mathbb{R}^{+}$for a wide class of initial and boundary values, where $F$ and $G$ are smooth functions so that the system has three rest points.

\section{INTRODUCTION}

In this paper we study the system

$$
u_{t}=u_{x x}+F(u, v), \quad v_{t}=G(u, v), \quad(x, t) \in D
$$

where $D=\mathbb{R} \times \mathbb{R}^{+}$or $D=\mathbb{R}^{+} \times \mathbb{R}^{+}$, with $G(u, v)=\gamma(u)(k(u)-v), u \geqslant 0, v \geqslant 0$ (see $[2,6])$ and we assume:

1. There is an interval $[a, b] \subseteq[0, \infty)$ such that $F(u, v)$ is analytic on $[a, b] \times[0, \infty)$, and $\gamma(u), k(u)$ are analytic and positive on $[a, b]$.

2. $F_{u}<0, F_{v}<0, G_{u}<0, G_{v}<0 \forall(u, v) \in[a, b] \times[0, \infty)$.

3. There exists a function $h(u)$, analytic and positive on $[a, b]$ such that $F(u, v)=0 \Leftrightarrow v=h(u)$.

4. The equation $h(u)=k(u)$ has exactly three roots, $u_{0}<u_{1}<u_{2}$ in $(a, b)$ such that: $h^{\prime}\left(u_{0}\right)<k^{\prime}\left(u_{0}\right), h^{\prime}\left(u_{1}\right)>k^{\prime}\left(u_{1}\right), h^{\prime}\left(u_{2}\right)<k\left(u_{2}\right)$.

5. $I\left(u_{2}\right)=\int_{u_{0}}^{u_{2}} F(u, k(u)) d u>0$.

If $v_{i}=h\left(u_{i}\right), i=1,2,3$, then, from the above assumptions we have:

(a) $h^{\prime}(u)<0, k^{\prime}(u)<0$ on $[a, b]$;

(b) $\left.\left(F_{u} G_{v}-F_{v} G_{u}\right)\right|_{\left(u_{i}, v_{i}\right)}>0, i=0,2$;

(c) $F(u, v)>0$ for $v<h(u)$, $G(u, v)>0$ for $v<k(u), \forall u \in(a, b)$.

Received 26 November 1991

Research partially supported by Fondecyt, grant No. 0244-91, and Universidad Santa Maria, grant No. 92.12.07.

Copyright Clearance Centre, Inc. Serial-fee code: 0004-9729/92 \$A2.00+0.00. 
This system appears in equations of nerve conduction models, chemical reaction et cetera $[2,3]$.

We investigate the asymptotic behaviour, as $t \rightarrow \infty$, of solutions of (1.1) under the initial-boundary conditions:

$$
\begin{aligned}
& u(x, 0)=f(x) \quad v(x, 0)=g(x), \quad x \geqslant 0 \\
& u(0, t)=h(t), \quad t \geqslant 0
\end{aligned}
$$

Since $u$ and $v$ represent chemical concentration, it is natural to impose the conditions

$$
u_{0} \leqslant f(x), \quad h(t) \leqslant u_{2}, \quad v_{2} \leqslant g(x) \leqslant v_{0}, \quad x \geqslant 0, \quad t \geqslant 0 .
$$

We are interested in the stability of the equilibrium states $\left(u_{i}, v_{i}\right), i=0,1,2$, and to show that $\left(u_{0}, v_{0}\right),\left(u_{2}, v_{2}\right)$ are stable states while $\left(u_{1}, v_{1}\right)$ is unstable. So we may expect to have a threshold phenomenon in this case.

The main tool to be used is the following comparison principle: let $N(u, v)=$ : $u_{t}-u_{x x}-F(u, v), M(u, v)=: v_{t}-G(u, v)$.

Comparison Principle. (see [5]) Let $U(x, t)=(u(x, t), v(x, t)), \bar{U}(x, t)=$ $(\bar{u}(x, t), \bar{v}(x, t))$ be bounded and of class $C^{2}$ with $N(U) \leqslant 0, M(U) \geqslant 0, N(\bar{U}) \geqslant 0$ and $M(\bar{U}) \leqslant 0$ on $\mathbb{R}^{+} \times \mathbb{R}^{+}$. If $u(x, 0) \leqslant \bar{u}(x, 0), v(x, 0) \geqslant \bar{v}(x, 0)$ and $u(0, t) \leqslant$ $\bar{u}(0, t) \forall x \in \mathbb{R}^{+}, \forall t \in \mathbb{R}^{+}$, then

$$
u(x, t) \leqslant \bar{u}(x, t) \quad \text { and } \quad \bar{v}(x, t) \leqslant v(x, t), \quad \forall(x, t) \in \mathbb{R}^{+} \times \mathbb{R}^{+}
$$

REMARK. A similar comparison principle holds for the pure initial value problem (1.1)(1.2) (see [5]).

In Section 2 of this paper we analyse the stability of the rest points for the pure initial value problem, while in Section 3 we study its stability for the initial boundary value problem.

\section{INITIAL VALUE PROBLEM}

A steady state solution of $(1.1)$ in $(a, b)$ is a solution $(\tau(x), s(x))$ of the equation

$$
\tau^{\prime \prime}(x)+F(\tau(x), k(\tau(x)))=0
$$

where $s(x)=k(\tau(x))$.

We require the following lemma. 
Lemma 1. Let $(\tau(x), k(\tau(x))) \in\left[u_{0}, u_{2}\right] \times\left[v_{2}, v_{0}\right]$ be a steady state solution of $(1.1)$ on $(a, b)$ with $-\infty \leqslant a<b \leqslant+\infty$. If $a>-\infty$ we suppose that $\tau(a)=u_{0}, k(\tau(a))=v_{0}$, and if $b<\infty$ suppose that $\tau(b)=u_{0}, k(\tau(b))=v_{0}$. If $\left(w_{1}(x, t), w_{2}(x, t)\right)$ is a solution of (1.1) with initial conditions:

$$
\begin{aligned}
& w_{1}(x, 0)= \begin{cases}\tau(x) & x \in(a, b), \\
u_{0} & \text { otherwise, }\end{cases} \\
& w_{2}(x, 0)= \begin{cases}k(\tau(x)) & x \in(a, b), \\
v_{0} & \text { otherwise, }\end{cases}
\end{aligned}
$$

then $w_{1}(x, t)$ (respectively $w_{2}(x, t)$ ) is nondecreasing (nonincreasing) in $t$, for each $x$, fixed. Furthermore

$$
\lim _{t \rightarrow \infty}\left(w_{1}(x, t), w_{2}(x, t)\right)=(q(x), r(x))
$$

uniformly in each $x$-bounded interval, where $q(x)$ (respectively $r(x)$ ) is the smallest (biggest) steady state solution of $(1.1)$ in $\left[u_{0}, u_{2}\right]$ (respectively $\left[v_{2}, v_{0}\right]$ ) in the sense that:

$$
q(x) \geqslant \tau(x), \quad r(x) \leqslant k(\tau(x)) \quad \text { in }(a, b) .
$$

Proof: The proof of this lemma mimics that in [1], for a single equation, and we have omitted it for sake of brevity.

REMARK. Since $I\left(u_{2}\right)>0$, there exists $K \in\left[u_{1}, u_{2}\right]$ such that $I(K)=0$. Moreover $I(q(x))>0$ and $I^{\prime}(q)=F(q, k(q))>0$ for $q \in\left(K, u_{2}\right)$. Then for any $\beta \in\left(K, u_{2}\right)$ the solution $q_{\beta}(x)$ of (2.1) with first integral $q^{\prime 2}+2 I(q)=2 I(\beta)$ such that $q(0)=u_{0}$, $q^{\prime}(0)=\sqrt{2 I(\beta)}$ satisfy: $q_{\beta}>u_{0}$ on $\left(0, b_{\beta}\right) q_{\beta}(0)=q_{\beta}\left(b_{\beta}\right)=u_{0}$, and $q_{\beta}(x) \leqslant$ $q_{\beta}\left(b_{\beta} / 2\right)=\beta$ on $\left[0, b_{\beta}\right]$ where

$$
b_{\beta}=2 \int_{u_{0}}^{\beta}\{2(I(\beta)-I(u))\}^{-1 / 2} d u
$$

Then with this remark, we can state:

Theorem 2.1. Let $(u(x, t), v(x, t))$ be a solution of $(1.1)$ on $\mathbb{R} \times \mathbb{R}^{+}$such that $I\left(u_{2}\right)>0$. If for some $\beta \in\left(K, u_{2}\right)$ and some $x_{0}$ so that $u(x, 0) \geqslant q_{\beta}\left(x-x_{0}\right)$, $v(x, 0) \leqslant k\left(q_{\beta}\left(x-x_{0}\right)\right)$ on $\left(x_{0}, x_{0}+b_{\beta}\right)$, then we have

$$
\lim _{t \rightarrow \infty}(u(x, t), v(x, t))=\left(u_{2}, v_{2}\right)
$$

Proof: Since $\left(u_{0}, v_{0}\right)$ is a solution of $(1.1)$ and $u(x, 0) \geqslant u_{0} v(x, 0) \leqslant v_{0}$, $\forall x \in \mathbb{R}$. Then by the comparison theorem we have that $u(x, t) \geqslant u_{0}, v(x, t) \leqslant v_{0}$, 
$\forall(x, t) \in \mathbb{R} \times \mathbb{R}^{+}$. Let $u_{1}(x, t), v_{1}(x, t)$ be a solution of (1.1) such that:

$$
\begin{aligned}
& u_{1}(x, 0)= \begin{cases}q_{\beta}\left(x-x_{0}\right) & \text { on }\left(x_{0}, x_{0}+b_{\beta}\right) \\
u_{0} & \text { otherwise, }\end{cases} \\
& v_{1}(x, 0)= \begin{cases}k\left(q_{\beta}\left(x-x_{0}\right)\right) & \text { on }\left(x_{0}, x_{0}+b_{\beta}\right) \\
v_{0} & \text { otherwise. }\end{cases}
\end{aligned}
$$

Then by Lemma 1 there exists a stationary solution $\left(\tau_{1}(x), s_{1}(x)\right)$ so that

$$
\lim _{t \rightarrow \infty}\left(u_{1}(x, t), v_{1}(x, t)\right)=\left(\tau_{1}(x), s_{1}(x)\right)
$$

uniformly in each $x$-bounded interval, where for some $x_{0}$ :

$$
\tau_{1}(x) \geqslant q\left(x-x_{0}\right), \quad s_{1}(x) \leqslant k\left(q_{\beta}\left(x-x_{0}\right)\right) \quad \text { in }\left(x_{0}, x_{0}+b_{\beta}\right) .
$$

Then by the hypothesis on the initial conditions and the comparison principle we have: $u(x, t) \geqslant u_{1}(x, t)$ and $v(x, t) \leqslant v_{1}(x, t)$. On the other hand $u_{0} \leqslant u(x, t) \leqslant u_{2}$, $v_{2} \leqslant v(x, t) \leqslant v_{0}$ so it is sufficient to prove that $\tau_{1}(x)=u_{2}$ and $s_{1}(x)=k\left(u_{2}\right)=v_{2}$. Let us suppose that $\tau_{1}(x)<u_{2}$. Since $\tau_{1}(x)$ satisfies: $\tau_{1}^{\prime 2}(x) / 2+I\left(\tau_{1}(x)\right)=P$ for some constant $P \geqslant I(\beta)>0$, we may assume that there exists $x_{1}$ such that $\tau_{1}\left(x_{1}\right)=$ $\gamma \in\left[u_{0}, u_{2}\right)$ so we have:

$$
x-x_{1}=\mp \int_{\tau_{1}}^{\gamma}\{2(P-I(u))\}^{-1 / 2} d u
$$

where the sign depends on the sign of $\tau_{1}^{\prime}(x)$. From this it follows that for finite $x^{*}$, $\tau\left(x^{*}\right)=u_{0}$ with $\tau^{\prime}\left(x^{*}\right) \neq 0$, hence $\tau_{1}(x)$ takes values smaller than $u_{0}$, which is not possible. Therefore $\tau_{1}(x)=u_{2}$ and so

$$
\lim _{t \rightarrow \infty}(u(x, t), v(x, t))=\left(u_{2}, k\left(u_{2}\right)\right)=\left(u_{2}, v_{2}\right)
$$

REMARK. In order to study the stability of the equilibrium point $\left(u_{0}, v_{0}\right)$ and to estimate "how big" the initial condition must be to obtain the stability of this point, we use contracting rectangles for the vector field

$$
B(p, r)=\left(F\left(p+u_{0}, v_{0}-r\right), G\left(p+u_{0}, v_{0}-r\right)\right)^{t},
$$

of equation (1.1), in the following sense. 
DEFINITION: A bounded convex set $R \subseteq \mathbb{R}^{2}$ is contracting for the vector field $H(p, r)$ if for any point $(p, r) \in \partial R$ and every outward unit normal $\vec{n}$ at $(p, r)$ : $H(p, r) \cdot \vec{n}<0$.

Theorem 2.2. Let $u(x, t), v(x, t)$ be a solution of (1.1) and let $R$ be the rectangle

$$
\begin{aligned}
& R=\left\{(u, v) \mid u_{0}-\varepsilon\right. \leqslant u \leqslant u_{1}-\varepsilon, v^{*} \leqslant v<v^{* *}, 0<\varepsilon<u_{1}-u_{0}, \\
&\left.v^{*}=\frac{1}{2}(h+k)\left(u_{1}-\varepsilon\right), v^{* *}=\frac{1}{2}(h+k)\left(u_{0}-\varepsilon\right)\right\} .
\end{aligned}
$$

If $(u(x, 0), v(x, 0))=U(x, 0) \in R, \forall x \in \mathbb{R}$ and $U(x, 0)$ tend to $\left(u_{0}, v_{0}\right)$ as $x \rightarrow \infty$, then there exist positive constants $c, K$ such that:

$$
\left\|\left(u(x, t)-u_{0}, v_{0}-v(x, t)\right)\right\|_{\infty} \leqslant K e^{-c t} \forall t>0 .
$$

PROOF: It is easy to check that $R$ is a contracting set for the given vector field and that $\tau R=\{(\tau p, \tau r) \mid(p, r) \in R\}$ is a contraction of $R$ about $\left(u_{0}, v_{0}\right)$, for any $\tau \in(0,1]$. Since $U(x, 0) \in R \quad \forall x \in \mathbb{R}$, there exists $\tau \in(0,1]$ such that $U(x, 0) \in \tau R$. If $L$ is the largest side of the rectangle $\tau R$ then by the basic lemma of Rauch and Smoller [4, Lemma 3.8] there exists $s \in \mathbb{R}^{+}$such that the upper Dini derivative satisfies:

$$
\bar{D} q_{\tau R}(U(, t)) \leqslant-(s / L) q_{\tau_{R}}(U(, t)) ; \quad q_{\tau R}(U(x, 0))=\tau \leqslant 1 .
$$

where

$$
q_{R}(p(, t), r(, t))=\operatorname{Sup}_{x \in \mathbb{R}} \operatorname{Inf}\{\tau \geqslant 0 \mid(p(x, t), r(x, t)) \in \tau R\}
$$

Then

$$
q_{\tau R}((U(, t))) \leqslant e^{-(\bullet / L) t} q_{\tau R}(U(, 0))<K e^{-(\bullet / L) t}
$$

and the theorem follows.

REMARKs. (1) Since $\varepsilon>0$ is arbitrary, we may choose it sufficiently small so that $U(x, 0) \in R$ for all $x \in \mathbb{R}$. Hence letting $\varepsilon \rightarrow 0^{+}$we see that the initial conditions are bounded by

$$
u_{0} \leqslant u(x, t) \leqslant u_{1}, \quad v_{1} \leqslant v(x, t) \leqslant v_{0} .
$$

(2) In the same manner we may prove that the steady state $\left(u_{2}, v_{2}\right)$ is asymptotically exponentially stable with domain of stability given by $u_{1} \leqslant u(x, 0) \leqslant u_{2}$, $v_{2} \leqslant v(x, 0) \leqslant v_{1}$.

(3) From the above, we see that the steady state $\left(u_{1}, v_{1}\right)$ is unstable, that is, it is a threshold point. 


\section{INITIAL-BOUNDARY VALUE PROBLEM}

Let us consider the boundary value problem

$$
\begin{array}{lll}
u_{t}=u_{x x}+F(u, v), & v_{t}=G(u, v), & x>0, t>0 \\
u(x, 0)=u_{0}, & v(x, 0)=v_{0}, & x>0 \\
u(0, t)=h(t) \in\left[u_{0}, u_{2}\right] & & \forall t>0 .
\end{array}
$$

An analogous lemma to Lemma 1, reads:

Lemma 2. Let $(\tau(x), s(x))$ be a stationary solution of $(3.1)$ in $(a, b)$ with $a>0$ and let $\tau(\dot{a})=\tau(b)=u_{0}, s(a)=s(b)=v_{0}$. Let $\left(w_{1}(x, t), w_{2}(x, t)\right)$ be a solution of (3.1) with initial-boundary conditions

$$
\begin{aligned}
& w_{1}(x, 0)= \begin{cases}\tau(x) & \text { in }(a, b) \\
u_{0} & \text { on } \mathbb{R}^{+} \backslash(a, b),\end{cases} \\
& w_{2}(x, 0)= \begin{cases}s(x) & \text { in }(a, b) \\
v_{0} & \text { on } \mathbb{R}^{+} \backslash(a, b)\end{cases} \\
& w_{1}(x, 0)=\psi(t) \text { on } \mathbb{R}^{+} \text {. }
\end{aligned}
$$

Suppose that $\psi(t)$ in nondecreasing and $\psi(0)=u_{0}$ with $\psi(t) \in\left[u_{0}, u_{2}\right]$. Then $w_{1}(x, t)$ (respectively $w_{2}(x, t)$ ) is non-decreasing (non-increasing) in $t$, for each $x$ fixed. Furthermore

$$
\lim _{t \rightarrow \infty}\left(w_{1}(x, t), w_{2}(x, t)\right)=(q(x), r(x))
$$

uniformly in each $x$-bounded interval, where $(q(x), \tau(x))$ is a steady state solution of (3.1) and they satisfy:

$$
q(0) \geqslant \lim _{t \rightarrow \infty} \psi(t) \text { and } q(x) \geqslant \tau(x), r(x) \leqslant s(x) \quad \text { in }(a, b) .
$$

Remark. Consider the problem

$$
\tau^{\prime \prime}(x)+F(\tau(x), k(\tau(x)))=0 \text { on } \mathbb{R}^{+}, \quad \tau(0)=\beta .
$$

This equation has a unique solution on $\left[u_{0}, u_{2}\right]$ for each $\beta \in\left(K, u_{2}\right]$, which converges to $u_{2}$ as $x \rightarrow \infty$, and it has two solutions for $\beta \in\left[u_{0}, K\right)$, one of which converges to $u_{0}$ as $x \rightarrow \infty$.

Theorem 3.1. Let $(u(x, t), v(x, t))$ be a solution of $(3.1)-(3.3)$ and let $\left(p_{\beta}(x), k\left(p_{\beta}(x)\right)\right)$ be a steady state solution of (3.1) such that $p_{\beta}(0)=\beta, p_{\beta}^{\prime}(0)=0$, for some $\beta \in\left[K, u_{2}\right]$. For any $\beta \in\left(K, u_{2}\right)$ there exist positive numbers $a_{\beta}$ and 
$t_{\beta}$ such that $p_{\beta}\left( \pm a_{\beta}\right)=u_{0}$ and if $h(t) \geqslant \beta, t \in\left(t_{1}, t_{1}+t_{\beta}\right)$, some $t_{1}>0$. Then the solution $(u(x, t), v(x, t))$ of (3.1) satisfies $u\left(x, t_{1}+t_{\beta}\right) \geqslant p_{\beta}\left(x-a_{\beta}-1\right)$, $v\left(x, t_{1}+t_{\beta}\right) \leqslant k\left(p_{\beta}\left(x-a_{\beta}-1\right)\right)$, for all $x \in\left(1,1+2 a_{\beta}\right)$, and

$$
\lim _{x \rightarrow \infty} \lim _{t \rightarrow \infty}(\operatorname{Inf} u(x, t), \operatorname{Sup} v(x, t))=\left(u_{2}, v_{2}\right) .
$$

Proof: Since $\left(u_{0}, v_{0}\right)$ is a solution of (1.1)-(1.3) then by the comparison theorem: $u(x, t) \geqslant u_{0}, v(x, t) \leqslant v_{0}$ on $\mathbb{R}^{+} \times \mathbb{R}^{+}$. Define $s(t)$ as a smooth and nondecreasing function so that $s(t)=u_{0}$, for $t \in(-\infty, 0)$, and $s(t)=\beta$, for $t \in(1,+\infty)$. Let $\left(w_{1}(x, t), w_{2}(x, t)\right)$ be a solution of (3.1)-(3.3) on $\mathbb{R}^{+} \times \mathbb{R}^{+}$such that $w_{1}(0, t)=$ $s(t), t \in \mathbb{R}^{+}$. Then it is well-known that the solution $\left(w_{1}(x, t), w_{2}(x, t)\right)$ converges, uniformly in $x$, as $t \rightarrow \infty$, to a steady state solution of (3.1)-(3.3), $(q(x), k(q(x)))$ with $q(0) \geqslant \lim _{t \rightarrow \infty} s(t)=\beta$. Since $\beta>K$ the problem:

$$
q^{\prime \prime}(x)+F(q(x), k(q(x)))=0, \quad x \in \mathbb{R}^{+}, q(0)=\beta .
$$

has a unique solution $q(x)$ such that $q(x) \rightarrow u_{2}, k(q(x)) \rightarrow v_{2}$ as $x \rightarrow \infty$. Furthermore, from the phase portrait of (3.7) we learn that there exist a number $a_{\beta}$ and a solution $p_{\beta}(x)$ defined on $\left(0, a_{\beta}\right)$ such that $p_{\beta}(0)=p_{\beta}(a)=u_{0}$ and $p_{\beta}(x) \leqslant p_{\beta}(a / 2)=\tau$ on $\left(0, a_{\beta}\right)$. Thus $p_{\beta}(x-1)<s(x)$ and $k\left(p_{\beta}(x-1)\right)>k(s(x))$ on $\left(1,1+a_{\beta}\right)$. Since the convergence of $\left(w_{1}, w_{2}\right)$ to $(s(x), k(s(x)))$ is uniform on $\left[1,1+a_{\beta}\right]$, there exist a time $t_{\beta}$ for which, on $\left[1,1+a_{\beta}\right]$, we have:

$$
w_{1}\left(x, t_{\beta}\right) \geqslant p_{\beta}(x-1), \quad w_{2}\left(x, t_{\beta}\right) \leqslant k\left(p_{\beta}(x-1)\right) .
$$

Then, by the comparison theorem, we have:

$$
u\left(x, t+t_{1}\right) \geqslant w_{1}(x, t), \quad v\left(x, t+t_{1}\right) \leqslant w_{2}(x, t) \text { on } \mathbb{R}^{+} \times\left[0, t_{\beta}\right] .
$$

Therefore $\lim _{t \rightarrow \infty} \operatorname{Inf} u(x, t)$ (respectively, $\lim _{t \rightarrow \infty} \operatorname{Sup} v(x, t)$ ) is bounded below (respectively, above) by a stationary solution $s_{1}(x)$ (respectively, $k\left(s_{1}(x)\right)$ ) of (3.7), such that

$$
s_{1}(x) \geqslant p_{\beta}(x-1), \quad k\left(s_{1}(x)\right) \leqslant k\left(p_{\beta}(x-1)\right) \text { on }\left[1,1+a_{\beta}\right] .
$$

In particular, $s_{1}\left(x+a_{\beta} / 2\right) \geqslant \beta>K$. Hence $\lim _{x \rightarrow \infty}\left(s_{1}(x), k\left(s_{1}(x)\right)\right)=\left(u_{2}, v_{2}\right)$ and the theorem follows.

TheOREM 3.2. Let $(u(x, t), v(x, t))$ be a solution of (3.1)-(3.3) and let $\beta=$ Sup $h(t)<K$. Then $u(x, t) \leqslant q_{\beta}(x), v(x, t) \geqslant p_{\beta}(x)$. In particular,

$$
\lim _{x \rightarrow \infty} \lim _{t \rightarrow \infty}(\operatorname{Sup} u(x, t), \operatorname{Inf} v(x, t))=\left(u_{0}, v_{0}\right)
$$


where $\left(q_{\beta}(x), p_{\beta}(x)\right)$ is a steady state solution of (3.1).

Proof: From the remark we have $\left(q_{\beta}(x), p_{\beta}(x)\right) \rightarrow\left(u_{0}, v_{0}\right)$ as $x \rightarrow \infty$ and the result follows directly from the comparison principle, because $u(0, t)=h(t)<\beta$, $u(x, 0)=u_{0} \leqslant q_{\beta}(x), v(x, 0)=v_{0} \geqslant p_{\beta}(x) \forall x \in \mathbb{R}^{+} .\left(\beta<K\right.$ implies $u_{0}<q_{\beta}(x)<$ $K)$.

\section{REFERENCES}

[1] D.G. Aronson and H.F. Weinberger, Nonlinear diffusion in population genetics, combustion and nerve conduction, Lecture Notes in Mathematics 446 (Springer-Verlag, New York, 1975).

[2] G.A. Klaasen and W. Troy, 'The stability of travelling wave front solutions of reaction diffusion system', SIAM J. Appl. Math. 41 (1981), 145-167.

[3] J.D. Murray, Mathematical biology, Biomathematics 19 (Springer-Verlag, Berlin, Heidelberg, New York, 1989).

[4] J. Rauch and J. Smoller, 'Qualitative theory of the FitzHugh-Nagumo equations', Adv. in Math. 27 (1978), 12-44.

[5] E. Tuma, 'Comparison principles for strongly coupled reaction diffusion equations in unbounded domains', Proc. Roy. Soc. Edinburgh 110 (1988), 311-319.

[6] E. Tuma and C.M. Blazquez, 'The stability of travelling wave front solutions in an initial-boundary reaction-diffusion system', J. Mech. App. Math. (to appear).

Department of Mathematics

Santa Maria University

PO Box 110-V

Valparaiso

Chile 